\title{
Enhanced Aedes spp. surveillance across jurisdictions in Arizona's border region
}

\author{
Mariana G. Casal ${ }^{\star 1}$, Nicolette Dent ${ }^{1}$, Jose Arriola ${ }^{2}$, Victor Dominguez ${ }^{2}$, Elizabeth Lueck ${ }^{3}$, \\ George Gentzsch ${ }^{4}$, Kathleen Walker ${ }^{5}$, Shelly Jacobs ${ }^{2}$ and Robert Guerrero'
}

${ }^{1}$ Office of Border Health, Arizona Department of Health Services, Tucson, AZ, USA; ${ }^{2}$ Santa Cruz County Health Department, Nogales, AZ, USA; ${ }^{3}$ Cochise County Health Department, Bisbee, AZ, USA; ${ }^{4}$ US Costums and Border Protection, Tucson, AZ, USA; ${ }^{5}$ University of Arizona Department of Entomology, Tucson, AZ, USA

\section{Objective}

This surveillance project aims to increase and broaden coverage of Aedes spp. ovitrap locations in Arizona's U.S.-Mexico border region through interagency collaboration.

\section{Introduction}

As part of a statewide effort to enhance surveillance for Aedes spp. mosquitoes $(1,2)$ the Office of Border Health $(\mathrm{OBH})$ took the lead in providing technical assistance on surveillance in counties bordering Mexico. In 2016, OBH sought ways to enhance surveillance in a wider geographic area. Trap locations closer to the border were established as a priority, given high amount of traffic across the international line, high border Aedes mosquito activity, and native cases of dengue reported at the border in Mexico.

\section{Methods}

The Arizona Office of Border Health partnered with U.S. Customs and Border Protection to select possible locations for ovitrapping near the border. Border Patrol Health and Safety Tucson coordination accompanied $\mathrm{OBH}$ and preparedness staff on three occasions to scout areas around pre-selected border patrol facilities. County, and border patrol staff contributed to trap maintenance. BIDS provided technical assistance to identify positive traps, collected data for reporting to the state, and collaborated with experts at the University of Arizona entomology department to verify results and identify Aedes spp.

\section{Results}

Out of 15 border patrol stations within border lands in Santa Cruz County, and Cochise County, OBH epidemiologist considered 10 viable trapping sites. Two facilities were eventually eliminated because of logistical challenges. OBH visited eight facilities and selected five locations within five miles of the U.S. -Mexico border and two located less than 30 miles from the border. OBH epidemiologists inspected sites for potential mosquito habitat and set ovitraps low to the ground in areas protected from rain. Some facilities had areas of standing water discovered in unused tires, truck-washing stations, heavy-lifting equipment, and natural washes. Border Patrol staff complained of mosquito activity around some of the stations. After inspection OBH set an average of three traps at each site. One site had evidence of mosquito larvae activity.

\section{Conclusions}

Border patrol facilities offer ideal trap locations given their proximity to the international line. Secure facilities offer extra protection for traps against tampering. The partnership across local, state, tribal, and federal lines allowed Arizona Office of Border Health to expand surveillance locations, allowing two jurisdictions to set the first Aedes-specific traps since Arizona began the 2016 campaign, "Fight the Bite."

\section{Keywords}

Aedes mosquito; ovitraps; inter-agency collaboration; mosquito surveillance

\section{References}

1.Fredericks, A. C., \& Fernandez-Sesma, A. (2014). THE BURDEN OF DENGUE AND CHIKUNGUNYA WORLDWIDE: IMPLICATIONS FOR THE SOUTHERN UNITED STATES AND CALIFORNIA. Annals of Global Health,80(6), 466-475. http://doi.org/10.1016/j. aogh.2015.02.006

2.Rafael Moreno-Sanchez, Mary Hayden, Craig Janes, Geoffrey Anderson, A web-based multimedia spatial information system to document Aedes aegypti breeding sites and dengue fever risk along the US-Mexico border, Health \& Place, Volume 12, Issue 4, December 2006, Pages 715-727, ISSN 1353-8292, http://dx.doi.org/10.1016/j. healthplace.2005.10.001.

\section{*Mariana G. Casal}

E-mail: mariana.casal@azdhs.gov 